\title{
Frailty, walking ability and self-rated health in predicting institutionalization: an 18-year follow-up study among Finnish community-dwelling older people
}

\author{
Anna Viljanen ${ }^{1,2}$ (D) Marika Salminen ${ }^{3,4} \cdot$ Kerttu Irjala $^{5} \cdot$ Päivi Korhonen $^{4} \cdot$ Maarit Wuorela $^{2,3} \cdot$ Raimo Isoaho $^{4,6}$. \\ Sirkka-Liisa Kivelä ${ }^{4,7} \cdot$ Tero Vahlberg $^{8} \cdot$ Matti Viitanen $^{2} \cdot$ Minna Löppönen $^{9} \cdot$ Laura Viikari $^{2,3}$
}

Received: 23 February 2020 / Accepted: 1 April 2020 / Published online: 18 April 2020

(c) The Author(s) 2020

\begin{abstract}
Background In clinical practice, there is a need for an instrument to screen older people at risk of institutionalization. Aims To analyze the association of frailty, walking-ability and self-rated health (SRH) with institutionalization in Finnish community-dwelling older people.

Methods In this prospective study with 10- and 18-year follow-ups, frailty was assessed using FRAIL Scale (FS) $(n=1087)$, Frailty Index (FI) $(n=1061)$ and PRISMA-7 $(n=1055)$. Walking ability was assessed as self-reported ability to walk $400 \mathrm{~m}$ $(n=1101)$. SRH was assessed by a question of general SRH $(n=1105)$. Cox regression model was used to analyze the association of the explanatory variables with institutionalization.

Results The mean age of the participants was 73.0 (range 64.0-97.0) years. Prevalence of institutionalization was 40.8\%. In unadjusted models, frailty was associated with a higher risk of institutionalization by FS in 10-year follow-up, and FI in both follow-ups. Associations by FI persisted after age- and gender-adjustments in both follow-ups. By PRISMA-7, frailty predicted a higher risk of institutionalization in both follow-ups. In unadjusted models, inability to walk $400 \mathrm{~m}$ predicted a higher risk of institutionalization in both follow-ups and after adjustments in 10-year follow-up. Poor SRH predicted a higher risk of institutionalization in unadjusted models in both follow-ups and after adjustments in 10-year follow-up.

Discussion Simple self-reported items of walking ability and SRH seemed to be comparable with frailty indexes in predicting institutionalization among community-dwelling older people in 10-year follow-up.

Conclusions In clinical practice, self-reported walking ability and SRH could be used to screen those at risk.
\end{abstract}

Keywords Association · Frailty $\cdot$ Institutionalization · Older people $\cdot$ Self-rated health $\cdot$ Walking ability

Electronic supplementary material The online version of this article (https://doi.org/10.1007/s40520-020-01551-x) contains supplementary material, which is available to authorized users.

Anna Viljanen

amvilj@utu.fi

1 Health Care Center, Municipality of Lieto, Hyvättyläntie 7, 21420 Lieto, Finland

2 Faculty of Medicine, Department of Clinical Medicine, Unit of Geriatrics, University of Turku, Turku City Hospital, Kunnallissairaalantie 20, 20700 Turku, Finland

3 Welfare Division, City of Turku, Yliopistonkatu 30, 20101 Turku, Finland

4 Faculty of Medicine, Department of Clinical Medicine, Unit of Family Medicine, University of Turku, Turku University Hospital, 20014 Turku, Finland
5 Faculty of Medicine, Department of Clinical Medicine, Unit of Clinical Chemistry, TYKSLAB, 20521 Turku, Finland

6 Social and Health Care, City of Vaasa, Ruutikellarintie 4, 65101 Vaasa, Finland

7 Faculty of Pharmacy, Division of Social Pharmacy, University of Helsinki, 00014 Helsinki, Finland

8 Institute of Clinical Medicine, Biostatistics, University of Turku, Turku, Finland

9 Social and Health Care for Elderly, City of Raisio, Sairaalakatu 5, 21200 Raisio, Finland 


\section{Introduction}

Frailty in older adults has been described as a phenotype [1] as well as an accumulation of deficits [2,3]. The prevalence of frailty increases with age $[4,5]$. Frailty is more prevalent in women than in men; however, women tolerate frailty better regarding the risk of adverse effects related to frailty $[4,6]$, such as a higher risk of mortality [5, 7], institutionalization [3, $8,9]$ and disability $[9,10]$.

Frailty is a dynamic state, and the possibility of slowing or reversing the cascade of decline in functional capacity with targeted multicomponent interventions has been proposed [11]. The total healthcare cost increase related to an individual transitioning into a frail state is remarkable, so the possibility of preventing or delaying the transition might lead to substantial cost-savings [12]. Physician's assessment of a patient's risk for adverse effects has been shown to be poorer than an objective measurement [13]. A simple measure of an older person's health in relation to future adverse effects is needed. The recommended $[11,14]$ screening tools for frailty include the Frail Scale (FS) [15, 16], which is simple to use and can be obtained from data already included in a comprehensive geriatric assessment (CGA) [10, 15]. Also PRISMA-7 is a fast and feasible tool for screening of frailty [17], and has been shown to have high sensitivity and moderate specificity in identifying frailty in community-dwelling older people $[18,19]$. The Rockwood Frailty Index is a broadly validated [20] frailty tool with good predictive capability $[4,21]$.

Slowness is a part of the classic phenotype definition of frailty, the Fried phenotype [1], and often measured as gait speed. Slow gait speed has been found to predict the inability to walk $400 \mathrm{~m}$ [22]. Gait speed declines with age and has been shown to predict mortality and institutionalization [23, 24]. Self-reported information on walking ability coincides well with the controlled ability to walk $400 \mathrm{~m}$ [25].

Self-rated health (SRH) has been shown to predict mortality [26, 27], institutionalization [28] and future health care expenditure [29] in an elderly population.

We have earlier demonstrated the predictive ability of three frailty tools (Frailty Index, Frail Scale and PRISMA-7) in relation to mortality in Finnish community-dwelling older adults [7]. The aim of the current study was to analyze whether the same frailty tools also predicted a higher risk of institutionalization among the same population during 10- and 18-year follow-ups, and also to analyze the predictive ability of selfreported ability to walk $400 \mathrm{~m}$ and self-rated health in association with institutionalization.

\section{Materials and methods}

\section{Study design and population}

This study is a part of the longitudinal epidemiological study carried out in the municipality of Lieto in southwest Finland [30]. All persons born in or prior to the year $1933(n=1596)$ were invited to participate in the baseline examination that took place between March 1998 and September 1999. Of those eligible, 63 died before they were examined and 273 refused or did not respond leaving 1260 (82\%) participants, 533 men and 727 women. An outlier, institutionalized before baseline at the age of 17, was excluded from the analyses leaving 1259 participants.

At baseline the study protocol consisted of an extensive interview on demographic and socioeconomic factors and health behavior, numerous laboratory tests, and a clinical examination including a comprehensive survey of the participants' medical records.

The follow-ups time were 10 and 18 years. Participants no longer living in Lieto at the end of $2016(n=86)$ were excluded from the present analyses predicting institutionalization, as it was not possible to ascertain whether they continued living at home or were institutionalized in another municipality. Also participants already living in institutional care $(n=67)$ at baseline were excluded from the institutionalization analyses. This left us with 1106 participants.

\section{Mortality}

Data from all participants who died before January 2017 were obtained from the official Finnish Cause of Death Registry using unique personal identification numbers.

\section{Institutionalization}

A total of 395 participants were institutionalized. Institutionalization was defined as permanent entry into a nursing home of which the data were gathered from the municipality's electronic patient record system and coded by month and year of entry.

\section{Frailty}

Frailty was characterized using three commonly used approaches: FRAIL scale (FS) [15, 16], Frailty Index (FI) $[2,3]$ and PRISMA-7 [31]. The information on participants' frailty status were gathered from baseline data.

The FS is a frailty screening tool based on the Fried frailty phenotype [1]. The original phenotype has been modified in several studies and has not lost its prognostic significance 
[32]. The FS uses five self-reported items [15, 16]. We used a slightly modified version of FS (Appendix 1). In addition, data of illnesses were gathered from patient records instead of self-reporting.

The FI is calculated as the proportion of individual's deficits in relation to the total amount of deficits chosen $[2,3]$. In this study we used an FI consisting of 36 deficits (Appendix 2). We used pre-described cut-points of FI $\leq 0.08$ for robust, FI $0.09-0.24$ for pre-frail and FI $\geq 0.25$ for frail [4].

PRISMA-7 includes seven self-reported items [31], and in this study we used a modified version (Appendix 3).

\section{Walking ability}

Walking ability was assessed by self-reported ability to walk $400 \mathrm{~m}$ independently (yes/no).

\section{Self-rated health}

The information about the participants' SRH was gathered at baseline by the question: "How would you describe your current state of health?" The answers were categorized into three groups: 'good', 'intermediate' and 'poor'.

\section{Ethics}

The study was conducted according to the guidelines of the Declaration of Helsinki. The Ethics Committee of the Hospital District of Southwest Finland approved the study protocol. Participants provided written informed consent for the study.

\section{Statistical analyses}

At baseline, differences between men and women were tested using the Chi squared test, Fisher's exact test or twosample $t$ test.

Hazard ratios (HRs) and their 95\% confidence intervals for institutionalization were calculated using Cox proportional hazard models. Proportional hazards assumption was tested using Martingale residuals. The follow-up periods were calculated from the baseline measurements to the end of the follow period of 10 and 18 years or to the death of the individual. We used death as a competitive factor in the analyses.

Firstly, unadjusted Cox regression analyzes were conducted for frailty tools, self-reported walking ability and SRH. Secondly, Cox regression analyzes for FI, FS, selfreported walking ability and SRH were adjusted for age and gender, which are items of PRISMA-7. The interaction between gender and all explanatory variables (frailty tools, walking ability and SRH) were included in Cox regression model. $P$ values less than 0.05 were considered statistically significant. All statistical analyzes were performed using SAS System for Windows, version 9.4 (SAS Institute Inc., Cary, NC, USA).

\section{Results}

\section{Baseline characteristics}

The mean age of the participants was 73.0 (SD 6.4, range 64.0-97.0) years and 57\% were women. More detailed baseline characteristics of 1106 study participants are shown in Table 1.

Three percent of participants were characterized as frail according to FS, 25\% according to FI and $18 \%$ according to PRISMA-7. Figure 1 shows a Venn diagram of overlapping participants categorized as frail by FS, FI and PRISMA-7. All of the participants categorized as frail by FS were also categorized frail by FI. Frailty (both pre-frailty and frailty) was more common in women than in men according to FI and FS, but according to PRISMA-7, more men were frail than women.

Only $8 \%$ ( $9 \%$ of women and $7 \%$ of men) of the participants were self-reportedly unable to walk $400 \mathrm{~m}$.

Table 1 Baseline characteristics of study participants $(n=1106)$

\begin{tabular}{ll}
\hline & $n(\%)$ \\
\hline Age, mean (SD), range & $73.0(6.4), 64.0-97.0$ \\
Age & \\
$64-74$ & $720(65)$ \\
$75-84$ & $312(28)$ \\
$\geq 85$ & $74(7)$ \\
Women & $633(57)$ \\
Living alone & $342(31)$ \\
Education & \\
More than basic ${ }^{\text {a }}$ or basic & $94(8)$ \\
Less than basic & $1012(92)$ \\
MMSE $<26$ & $270(24)$ \\
Body mass index, kg/m ${ }^{2}$ & \\
$<20$ & $42(4)$ \\
$20-24.9$ & $297(27)$ \\
$25-29.9$ & $495(45)$ \\
$30-34.9$ & $208(19)$ \\
$\geq 35$ & $61(6)$ \\
Number of prescribed medicines & \\
$<5$ & $833(75)$ \\
$5-7$ & $196(18)$ \\
$8-9$ & $54(5)$ \\
$\geq 10$ & $23(2)$ \\
\hline
\end{tabular}

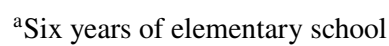




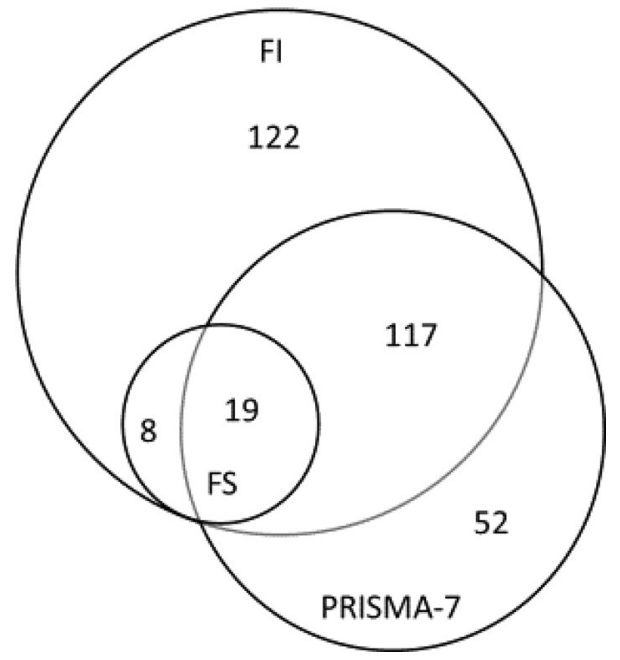

Fig. 1 Venn diagram of overlapping participants categorized as frail by FS $(n=27)$, FI $(n=266)$ and PRISMA-7 $(n=188)$

Altogether $14 \%(n=159)$ of participants rated their health as poor. The proportions of men and women in different groups of SRH (good, intermediate and poor) were similar: 40, 46 and $15 \%$ for men, and 41, 45 and $14 \%$ for women, respectively.

\section{Follow-up characteristics}

Of the participants, $212(31.1 \%)$ women and $130(26.5 \%)$ men were alive at the end of 2016.

Of the participants self-reportedly able to walk $400 \mathrm{~m}$ at baseline, $65 \%$ were still living at home, $12 \%$ institutionalized and $23 \%$ deceased after 10 years (at the end of 2008). After 18 years (at the end of 2016) the proportions were 28,28 and $44 \%$, respectively. Of the participants self-reportedly unable to walk $400 \mathrm{~m}, 10 \%$ were living at home, $43 \%$ institutionalized and $47 \%$ deceased after 10 years. After 18 years the proportions were 0,47 and 53\%, respectively.

Of the participants with good SRH at baseline, $72 \%$ were still living at home, $12 \%$ institutionalized and $16 \%$ deceased after 10 years. After 18 years, the proportions were 32, 29 and $39 \%$, respectively. Of the participants with poor SRH, $29 \%$ were living at home, $26 \%$ institutionalized and $45 \%$ deceased after 10 years. After 18 years, the proportions were 6,40 and $53 \%$, respectively.

\section{Prevalence of institutionalization}

When analyzing the proportion of participants institutionalized in the municipality of Lieto, we included participants who had been institutionalized before baseline. We only took into account the participants deceased by the end of 2016 , to count the proportion of participants institutionalized during their lifetime. This left us with 831 participants, of which 339 were institutionalized (40.8\%). There was a higher prevalence of institutionalization in women $(48.9 \%)$ than in men $(30.2 \%)$.

The mean age of the participants at the time of institutionalization and the mean time spent living in an institution are shown in Table 2.

\section{Cox models for frailty and institutionalization}

During the 10-year follow-up, both being frail and pre-frail according to FS and FI were associated with a higher risk of institutionalization in unadjusted Cox regression models (Table 3). After age- and gender-adjusted (items of PRISMA-7) analyses for FS and FI, the associations persisted for FI; pre-frailty according to FS also remained significantly related to higher risk of institutionalization. Also using the binary (robust or frail) PRISMA-7, being frail was associated with a higher risk of institutionalization during the 10-year follow-up.

During the 18-year follow-up, being pre-frail according to FS and both being pre-frail and frail according to FI were associated with a higher risk of institutionalization in unadjusted models (Table 4). After adjustments for age and gender, only being frail according to FI significantly associated with a higher risk of institutionalization. Also being frail according to PRISMA-7, was significantly related to a

Table 2 Age of study participants at time of institutionalization and mean time spent living in an institution

\begin{tabular}{|c|c|c|c|c|c|c|c|c|}
\hline & \multicolumn{4}{|c|}{ Mean age in years at time of institutionalization (SD) } & \multicolumn{4}{|c|}{ Mean time in years spent living in an institution (SD) } \\
\hline & Before baseline & $\begin{array}{l}\text { Baseline-January } \\
2008\end{array}$ & $\begin{array}{l}\text { January 2008- } \\
\text { January } 2017\end{array}$ & Study population & Before baseline & $\begin{array}{l}\text { Baseline- } \\
\text { January } 2008\end{array}$ & $\begin{array}{l}\text { January 2008- } \\
\text { January } 2017\end{array}$ & $\begin{array}{l}\text { Study } \\
\text { population }\end{array}$ \\
\hline Both $[n]$ & $81.6(7.0)[67]$ & $83.9(5.9)[160]$ & $87.4(5.3)[112]$ & $84.6(6.3)[339]$ & $5.2(3.8)$ & $3.2(2.7)$ & $1.7(1.5)$ & $3.1(2.9)$ \\
\hline Men $[n]$ & $81.1(6.6)[18]$ & $82.3(5.6)[54]$ & $85.4(5.0)[37]$ & $83.1(5.8)[109]$ & $4.5(3.6)$ & $2.7(2.6)$ & $1.5(1.4)$ & $2.6(2.7)$ \\
\hline Women $[n]$ & $81.8(7.1)[49]$ & $84.7(5.9)[106]$ & $88.4(5.1)[75]$ & $85.3(6.4)[230]$ & $5.4(3.9)$ & $3.5(2.8)$ & $1.9(1.5)$ & $3.4(3.3)$ \\
\hline
\end{tabular}


Table 3 Unadjusted and adjusted hazard ratios (HR) and their 95\% confidence intervals (CI) (in parentheses) of frailty indexes, self-reported ability to walk $400 \mathrm{~m}$ and self-rated health for institutionalization during the 10 -year follow-up

\begin{tabular}{|c|c|c|c|c|c|c|c|}
\hline & $\begin{array}{l}\text { Non-institu- } \\
\text { tionalized } n \\
(\%)\end{array}$ & $\begin{array}{l}\text { Institution- } \\
\text { alized } n \\
(\%)\end{array}$ & Deceased $n(\%)$ & Unadjusted HR (95\% CI) & $P$-value & Adjusted R $(95 \% \mathrm{CI})^{\mathrm{a}}$ & $P$-value \\
\hline \multicolumn{8}{|l|}{ FRAIL scale $(n=1087)$} \\
\hline Robust $(n=710)$ & $496(70)$ & $74(10)$ & $140(20)$ & 1 & & 1 & \\
\hline Pre-frail $(n=350)$ & $158(45)$ & $75(21)$ & $117(33)$ & $2.25(1.63-3.10)$ & $<.001$ & $1.48(1.04-2.10)$ & .030 \\
\hline Frail $(n=27)$ & $1(4)$ & $8(30)$ & $18(67)$ & $3.32(1.57-7.00)$ & .002 & $1.33(0.57-3.11)$ & .505 \\
\hline \multicolumn{8}{|l|}{ Frailty index $(n=1061)$} \\
\hline Robust $(n=197)$ & $164(83)$ & $8(4)$ & $25(13)$ & 1 & & 1 & \\
\hline Pre-frail $(n=596)$ & $402(67)$ & $64(11)$ & $130(22)$ & $2.74(1.32-5.69)$ & .007 & $2.32(1.12-4.79)$ & .023 \\
\hline Frail $(n=268)$ & $76(28)$ & $79(29)$ & $113(42)$ & $8.82(4.28-18.20)$ & $<.001$ & $4.50(2.11-9.63)$ & $<.001$ \\
\hline \multicolumn{8}{|l|}{ PRISMA-7 $(n=1055)$} \\
\hline Robust $(n=860)$ & $613(71)$ & $85(10)$ & $162(19)$ & 1 & & & \\
\hline Frail $(n=195)$ & $42(22)$ & $63(32)$ & $90(46)$ & $3.95(2.85-5.49)$ & $<.001$ & & \\
\hline \multicolumn{8}{|c|}{ Self-reported ability to walk $400 \mathrm{~m}(n=1101)$} \\
\hline Yes $(n=1011)$ & $653(65)$ & $123(12)$ & $235(23)$ & 1 & & 1 & \\
\hline No $(n=90)$ & $9(10)$ & $39(43)$ & $42(47)$ & $4.82(3.29-7.05)$ & $<.001$ & $2.06(1.25-3.41)$ & .005 \\
\hline \multicolumn{8}{|c|}{ Self-rated health $(n=1105)$} \\
\hline Good $(n=446)$ & $321(72)$ & $52(12)$ & $73(16)$ & 1 & & 1 & \\
\hline Moderate $(n=500)$ & $298(60)$ & $68(14)$ & $134(27)$ & $1.18(0.82-1.69)$ & .380 & $1.07(0.74-1.54)$ & .73 \\
\hline Poor $(n=159)$ & $46(29)$ & $42(26)$ & $71(45)$ & $2.51(1.67-3.77)$ & $<.0001$ & $1.59(1.00-2.53)$ & .05 \\
\hline
\end{tabular}

${ }^{a}$ Values are adjusted for age and gender (items included in PRISMA-7)

Table 4 Unadjusted and adjusted hazard ratios (HR) and their 95\% confidence intervals (CI) (in parentheses) of frailty indexes, self-reported ability to walk $400 \mathrm{~m}$ and self-rated health for institutionalization during the 18 -year follow-up

\begin{tabular}{|c|c|c|c|c|c|c|c|}
\hline & $\begin{array}{l}\text { Non-institu- } \\
\text { tionalized } n \\
(\%)\end{array}$ & $\begin{array}{l}\text { Institution- } \\
\text { alized } n \\
(\%)\end{array}$ & Deceased $n(\%)$ & Unadjusted HR (95\% CI) & $P$-value & Adjusted $^{\mathrm{a}} \mathrm{HR}(95 \% \mathrm{CI})$ & $P$-value \\
\hline \multicolumn{8}{|l|}{ FRAIL scale $(n=1087)$} \\
\hline Robust $(n=710)$ & $221(31)$ & $185(26)$ & $304(43)$ & 1 & & 1 & \\
\hline Pre-frail $(n=350)$ & $61(17)$ & $126(36)$ & $163(47)$ & $1.56(1.24-1.96)$ & $<.001$ & $1.22(0.96-1.55)$ & .110 \\
\hline Frail $(n=27)$ & $0(0)$ & $9(33)$ & $18(67)$ & $1.50(0.72-3.09)$ & .278 & $0.82(0.37-1.81)$ & .629 \\
\hline \multicolumn{8}{|c|}{ Frailty index $(n=1061)$} \\
\hline Robust $(n=197)$ & $73(37)$ & $36(18)$ & $88(45)$ & 1 & & 1 & \\
\hline Pre-frail $(n=596)$ & $192(32)$ & $159(27)$ & $245(41)$ & $1.56(1.10-2.21)$ & .013 & $1.38(0.98-1.97)$ & .068 \\
\hline Frail $(n=268)$ & $11(4)$ & $114(43)$ & $143(53)$ & $3.08(2.13-4.46)$ & $<.001$ & $2.00(1.34-2.97)$ & $<.001$ \\
\hline \multicolumn{8}{|l|}{ PRISMA-7 $(n=1055)$} \\
\hline Robust $(n=860)$ & $278(32)$ & $228(27)$ & $354(41)$ & 1 & & & \\
\hline Frail $(n=195)$ & $6(3)$ & $82(42)$ & $107(55)$ & $2.03(1.55-2.66)$ & $<.001$ & & \\
\hline \multicolumn{8}{|c|}{$\begin{array}{l}\text { Self-reported ability to walk } 400 \mathrm{~m} \\
(n=1101)\end{array}$} \\
\hline Yes $(n=1011)$ & $284(28)$ & $285(28)$ & $442(44)$ & 1 & & 1 & \\
\hline No $(n=90)$ & $0(0)$ & $42(47)$ & $48(53)$ & $2.31(1.59-3.36)$ & $<.001$ & $1.28(0.82-2.01)$ & .305 \\
\hline \multicolumn{8}{|c|}{ Self-rated health $(n=1105)$} \\
\hline Good $(n=446)$ & $143(32)$ & $128(29)$ & $175(39)$ & 1 & & 1 & \\
\hline Moderate $(n=500)$ & $133(27)$ & $136(27)$ & $231(46)$ & $0.96(0.76-1.22)$ & .75 & $0.91(0.72-1.16)$ & .461 \\
\hline Poor $(n=159)$ & $10(6)$ & $64(40)$ & $85(53)$ & $1.65(1.21-2.25)$ & .002 & $1.29(0.92-1.81)$ & .137 \\
\hline
\end{tabular}

${ }^{\text {a }}$ Values are adjusted for age and gender (items included in PRISMA-7) 

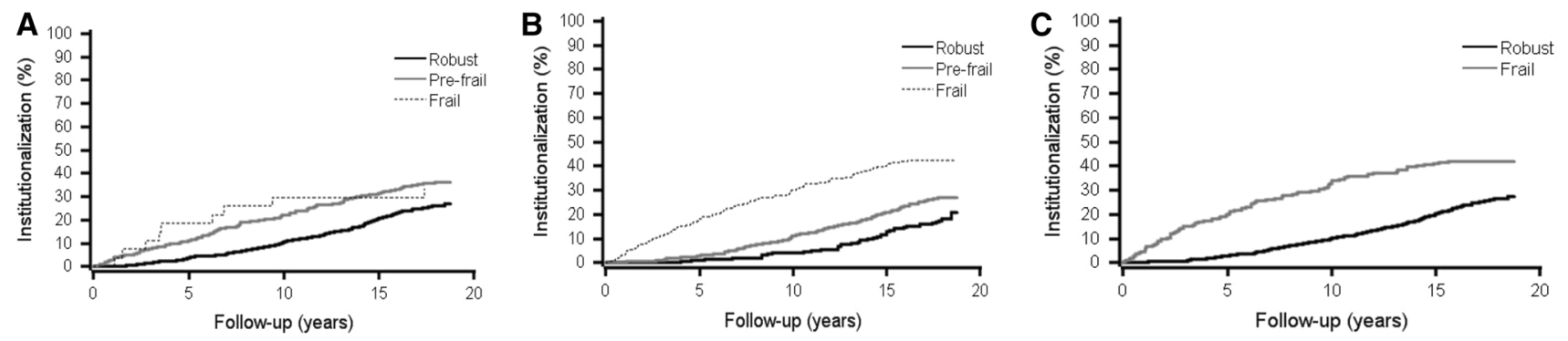

Fig. 2 Rates of institutionalization with death as a competing risk by Frail Scale (a), Frailty Index (b), and PRISMA7 (c) during the 18-year follow-up

higher risk of institutionalization. Figure 2 shows the rates of institutionalization by FI, FS and PRISMA-7 during the 18-year follow-up.

The association of frailty and institutionalization did not significantly differ between men and women either in 10- or 18-year follow-up by FI or PRISMA-7; using FS, being prefrail predicted a significantly higher risk of institutionalization in women (1.85 [1.41-2.43], $p<0.001)$, but not in men (0.98 [0.63-1.53], $p=0.930)$ during the 18 -year follow-up.

\section{Cox models for walking ability, self-rated health and institutionalization}

In an unadjusted model, the self-reported inability to walk $400 \mathrm{~m}$ and poor SRH were associated with a higher risk of institutionalization during both follow-ups. After adjustments, the associations persisted in 10-year follow-up. Figure 3 shows rates of institutionalization by self-reported walking ability and SRH during the 18-year follow-up.

No significant interaction was found between gender and self-reported walking ability in predicting the risk of institutionalization during either follow-ups. Poor SRH, instead, predicted a higher risk of institutionalization in women (1.85 [1.27-2.68], $p=0.001)$ but not in men (1.49 [0.86-2.60], $p=0.157$ ) during the 18 -year follow-up.

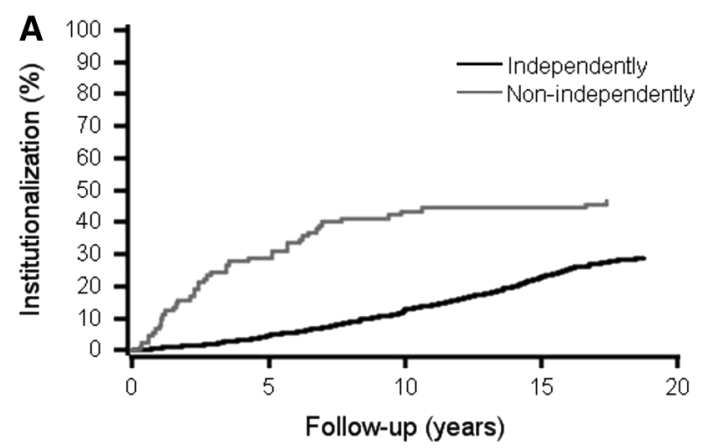

\section{Discussion}

In our study, the prevalence of frailty varied from $3 \%$ (according to FS) to 25\% (according to FI). Being frail according to FI and PRISMA-7 was associated with a higher risk of institutionalization in both follow-ups. Also being pre-frail according to FS was associated with a higher risk of institutionalization in 10-year follow-up. The self-reported inability to walk $400 \mathrm{~m}$ and poor SRH were associated with a higher risk of institutionalization in 10-year follow-up.

The current estimated prevalence of frailty in European adults aged 65 years and older is 10-20\% [33], but the estimate varies between population samples and the frailty tools used [4, 33]. Our findings were similar to previous studies regarding gender differences in categorizing participants as frail [6]. The current study also supports results of earlier studies showing that frailty tools differ in how they classify participants as frail and how they do not capture the same individuals, but still are capable of predicting adverse effects of frailty $[9,19]$.

Being frail according to FI was associated with a higher risk of institutionalization during both 10 and 18 year of follow-up also in adjusted analyses. Because of low

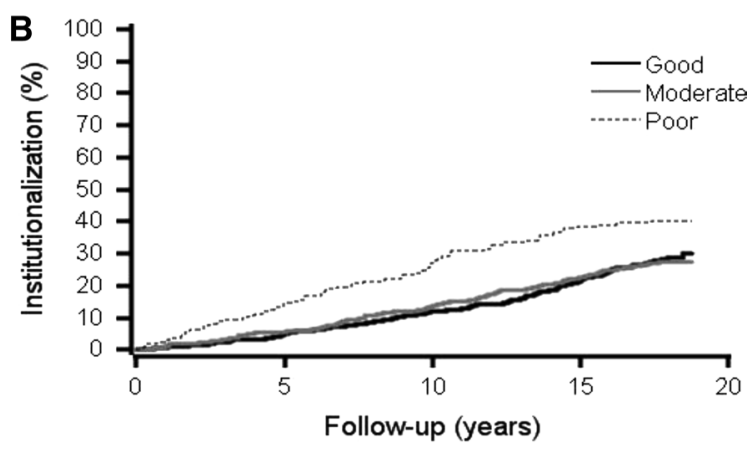

Fig. 3 Rates of institutionalization with death as a competing risk by self-reported ability to walk $400 \mathrm{~m}$ (a) and self-rated health (b) during the 18-year follow-up 
number of subjects categorized as frail, only being prefrail according to FS was related to higher risk of institutionalization during the 10-year follow-up. Frailty according to PRISMA-7 predicted a higher risk of institutionalization in both follow-ups. These results are in consistence with previous research $[4,8]$. In our previous study, we have also demonstrated that frailty according to all these three frailty tools predicted mortality up to 18 years [7].

In our study, the self-reported inability to walk $400 \mathrm{~m}$ at baseline was associated with a higher risk of institutionalization in 10-year follow-up. This is similar to previous research regarding walking ability [34]. In fact, the predictive ability of slow gait speed in regarding mortality and institutionalization has been found comparable to the FI [35]. Also similar to earlier research [28], we found that poor SRH at baseline was associated with a higher risk of institutionalization in 10-year follow-up. The finding is notable, since information about SRH is easy to acquire, with the exception of individuals with cognitive decline, whose assessment of their own health is not reliable due to loss of insight [36]. Poor SRH predicted a higher risk of institutionalization in women but not in men during the 18-year follow-up. Gender differences have been found earlier, but not always in the same direction [26, 27].

We also found that the mean age at the time of institutionalization has risen during the follow-up-period and the mean time spent living in an institution decreased. Women were institutionalized at a higher age than men and lived in an institution longer than men, reflecting that women seem to tolerate frailty and functional disabilities better than men $[4,6]$. Besides living longer, older people are staying longer at home than in previous years. These findings reflect the rising of the life expectancy of the Finnish older people during the follow-up period [37], and possibly also the change in the municipality's policy of the elderly care in which home care is preferred for many reasons, of which one is the high cost of institutional care. More women were institutionalized than men, due to the fact that women live longer, and perhaps do not have informal care available to them as often as to men. The role of formal or informal care at home, possibly affecting institutionalization, was not addressed in this study.

The strengths of our study are the large sample size and a long follow-up period enabling broad generalizability to the community-dwelling older population. We also used death as a competitive factor in our analyses. The frailty tools used were validated and commonly used, although the modified versions of FS and PRISMA-7 could have had an impact on our results. Especially, prevalence of frailty was low according to FS, and it's possible that the modifications used in our study underestimated the prevalence, and thus the association with institutionalization. The most obvious limitation to our study was that all studied explanatory variables were only assessed at baseline.

To conclude, a simple self-reported item, such as selfreported ability to walk $400 \mathrm{~m}$ and/or self-rated health could be used in the clinical setting instead of more time-consuming frailty tools, to screen for future risk of institutionalization, and identify older persons in need of a comprehensive evaluation and possible interventions.

Acknowledgements Open access funding provided by University of Turku (UTU) including Turku University Central Hospital. This study was financially supported by the municipality of Lieto, Turunmaa Duodecim Society, Turku University Department of Clinical Medicine, The Research Foundation for Laboratory Medicine and King Gustaf V's and Queen Victoria's Freemasons' Foundation. Open access funding provided by University of Turku (UTU) including Turku University Hospital.

\section{Compliance with ethical standards}

Conflict of interest On behalf of all authors, the corresponding author states that there is no conflict of interest.

Ethics approval The study was conducted according to the guidelines of the Declaration of Helsinki. The Ethics Committee of the Hospital District of Southwest Finland approved the study protocol. Participants provided written informed consent for the study.

Statement of human and animal rights All procedures performed in studies involving human participants were in accordance with the ethical standards of the institutional or national research committee and with the 1964 Helsinki declaration and its later amendments or comparable ethical standards. This article does not contain any studies with animals performed by any of the authors.

Informed consent Informed consent was obtained from all individual participants included in the study.

Open Access This article is licensed under a Creative Commons Attribution 4.0 International License, which permits use, sharing, adaptation, distribution and reproduction in any medium or format, as long as you give appropriate credit to the original author(s) and the source, provide a link to the Creative Commons licence, and indicate if changes were made. The images or other third party material in this article are included in the article's Creative Commons licence, unless indicated otherwise in a credit line to the material. If material is not included in the article's Creative Commons licence and your intended use is not permitted by statutory regulation or exceeds the permitted use, you will need to obtain permission directly from the copyright holder. To view a copy of this licence, visit http://creativecommons.org/licenses/by/4.0/.

\section{References}

1. Fried LP, Tangen CM, Walston J et al (2001) Frailty in older adults evidence for a phenotype. J Gerontol A Biol Sci Med Sci 56:M146-M157

2. Mitnitski AB, Graham JE, Mogilner AJ et al (2002) Frailty, fitness and late-life mortality in relation to chronological and biological age. BMC Geriatr 2:1 
3. Rockwood K, Howlett SE, MacKnight C et al (2004) Prevalence, attributes, and outcomes of fitness and frailty in communitydwelling older adults: report from the Canadian study of health and aging. J Gerontol Ser A Biol Sci Med Sci 59:1310-1317

4. Song X, Mitnitski A, Rockwood K (2010) Prevalence and 10-year outcomes of frailty in older adults in relation to deficit accumulation. J Am Geriatr Soc 58:681-687

5. Clegg A, Young J, Iliffe S et al (2013) Frailty in elderly people. Lancet 381:752-762

6. Gordon EH, Peel NM, Samanta M et al (2017) Sex differences in frailty: a systematic review and meta-analysis. Exp Gerontol 89:30-40

7. Salminen M, Viljanen A, Eloranta S et al (2019) Frailty and mortality: an 18-year follow-up study among Finnish community-dwelling older people. Aging Clin Exp Res. https://doi. org/10.1007/s40520-019-01383-4

8. Rockwood K, Mitnitski A, Song X et al (2006) Long-term risks of death and institutionalization of elderly people in relation to deficit accumulation at age 70. J Am Geriatr Soc 54:975-979

9. Gonzalez-Colaço Harmand M, Meillon C, Bergua V et al (2017) Comparing the predictive value of three definitions of frailty: results from the three-city study. Arch Gerontol Geriatr $72: 153-163$

10. Woo J, Leung J, Morley JE (2012) Comparison of frailty indicators based on clinical phenotype and the multiple deficit approach in predicting mortality and physical limitation. J Am Geriatr Soc 60:1478-1486

11. Dent E, Morley J, Cruz-Jentoft A et al (2019) Physical frailty: ICFSR international clinical practice guidelines for identification and management. J Nutr Health Aging 23:771-787

12. Hajek A, Bock J, Saum K et al (2018) Frailty and healthcare costs-longitudinal results of a prospective cohort study. Age Ageing 47:233-241

13. Jain R, Duval S, Adabag S (2014) How accurate is the eyeball test?: A comparison of Physician's subjective assessment versus statistical methods in estimating mortality risk after cardiac surgery. Circ Cardiovasc Qual Outcomes 7:151-156

14. Morley JE, Vellas B, van Abellan KG et al (2013) Frailty consensus: a call to action. J Am Med Direct Assoc 14:392-397

15. Morley JE, Malmstrom TK, Miller DK (2012) A simple frailty questionnaire (FRAIL) predicts outcomes in middle aged African Americans. J Nutr Health Aging 16:601-608

16. Lopez D, Flicker L, Dobson A (2012) Validation of the FRAIL scale in a cohort of older Australian women. J Am Geriatr Soc 60:171-173

17. Raîche M, Hébert R, Dubois MF (2007) PRISMA-7: a case-finding tool to identify older adults with moderate to severe disabilities. Arch Gerontol Geriatr 47:9-18.

18. Apóstolo J, Cooke R, Bobrowicz-Campos E et al (2017) Predicting risk and outcomes for frail older adults: an umbrella review of frailty screening tools. JBI Database Syst Rev Implement Rep 15:1154-1208

19. Clegg A, Rogers L, Young J (2015) Diagnostic test accuracy of simple instruments for identifying frailty in community-dwelling older people: a systematic review. Age Ageing 44:148-152

20. Mitnitski A, Song X, Skoog I et al (2005) Relative fitness and frailty of elderly men and women in developed countries and their relationship with mortality. J Am Geriatr Soc 53:2184-2189

21. Rockwood K, Mitnitski A (2007) Frailty in relation to the accumulation of deficits. J Gerontol 62:722-727
22. Chang M, Cohen-Mansfield J, Ferrucci L et al (2004) Incidence of loss of ability to walk 400 meters in a functionally limited older population. J Am Geriatr Soc 52:2094-2098

23. Rothman MD, Leo-Summers L, Gill TM (2008) Prognostic significance of potential frailty criteria. J Am Geriatr Soc 56:2211-2216

24. Studenski S, Perera S, Patel K et al (2011) Gait speed and survival in older adults. JAMA 305:50-58

25. Sayers SP, Brach JS, Newman AB et al (2004) Use of self-report to predict ability to walk 400 meters in mobility-limited older adults. J Am Geriatr Soc 52:2099-2103

26. Karen B, DeSalvo NB, Kristi R et al (2005) Mortality prediction with a single general self-rated health question - a meta-analysis. $\mathrm{J}$ Gen Intern Med

27. Jylhä M (2009) What is self-rated health and why does it predict mortality? Towards a unified conceptual model. Soc Sci Med 69:307-316

28. Gutman GM, Stark A, Donald A et al (2001) Contribution of selfreported health ratings to predicting frailty, institutionalization, and death over a 5-year period. Int Psychogeriatr 13:223-231

29. DeSalvo KB, Jones TM, Peabody J et al (2009) Health care expenditure prediction with a single item self-rated health measure. Med Care 47:440-447

30. Löppönen M, Räihä I, Isoaho R et al (2003) Diagnosing cognitive impairment and dementia in primary health care-a more active approach is needed. Age Ageing 32:606-612

31. Raîche M, Hébert R, Dubois M (2007) PRISMA-7: a case-finding tool to identify older adults with moderate to severe disabilities. Arch Gerontol Geriatr 47:9-18

32. Theou O, Cann L, Blodgett J et al (2015) Modifications to the frailty phenotype criteria: systematic review of the current literature and investigation of 262 frailty phenotypes in the survey of health, ageing, and retirement in Europe. Ageing Res Rev 21:78-94

33. O'Caoimh R, Galluzzo L, Rodríguez-Laso Á et al (2018) Prevalence of frailty at population level in European ADVANTAGE Joint action member states: a systematic review and meta-analysis. Annali dell'Istituto superiore di sanita 54:226-238

34. Hajek A, Brettschneider C, Lange $C$ et al (2015) Longitudinal predictors of institutionalization in old age. PLoS ONE 10:e0144203

35. Jung H, Jang I, Lee CK et al (2018) Usual gait speed is associated with frailty status, institutionalization, and mortality in community-dwelling rural older adults: a longitudinal analysis of the Aging Study of Pyeongchang Rural Area. Clin Interv Aging 13:1079-1089

36. Nielsen ABS, Siersma V, Waldemar G et al (2016) Poor self-rated health did not increase risk of permanent nursing placement or mortality in people with mild Alzheimer's disease. BMC Geriatr 16

37. Statistics Finland [Internet]; 2017 [cited 3.12.2019]. Available from: https://pxnet2.stat.fi/PXWeb/pxweb/en/StatFin/StatFin_ vrm_kuol/statfin_kuol_pxt_12ap.px/.

Publisher's Note Springer Nature remains neutral with regard to jurisdictional claims in published maps and institutional affiliations. 